EPJ Web of Conferences 93,01001 (2015)

DOI: 10.1051 /epjconf/20159301001

(C) Owned by the authors, published by EDP Sciences, 2015

\title{
Testing CVC and CKM Unitarity via superallowed nuclear beta decay
}

\author{
J.C. Hardy ${ }^{1, a}$, I.S. Towner ${ }^{1, b}$, H.I. Park ${ }^{1}$, V.E. lacob $^{1}$, L. Chen ${ }^{1}$, V. Horvat ${ }^{1}$, N. Nica ${ }^{1}$, and M. Bencomo ${ }^{1}$ \\ ${ }^{1}$ Cyclotron Institute, Texas A\&M University, College Station TX, 77843-3366
}

\begin{abstract}
Currently, the most restrictive test of the unitarity of the Cabibbo-Kobayashi-Maskawa (CKM) matrix is anchored by nuclear beta decay. Precise measurements of the $f t$-values for superallowed beta transitions between analog $0^{+}$states are used to determine $G_{\mathrm{V}}$, the vector coupling constant; this, in turn, yields $V_{u d}$, the up-down quark-mixing element of the CKM matrix. The determination of a transition's $f t$-value requires the measurement of three quantities: its $Q$ value, branching ratio and parent half-life. To achieve $0.1 \%$ precision on the final result, each of these quantities must be measured to substantially better precision, for which special techniques have had to be developed. A new survey and analysis of world data reveals that there are now fourteen such transitions with $f t$-values known to $\sim 0.1 \%$ precision or better, and that they span a wide range of nuclear masses, from ${ }^{10} \mathrm{C}$, the lightest parent, to ${ }^{74} \mathrm{Rb}$, the heaviest. Of particular interest is the recent completion of the first mirror pair of $0^{+} \rightarrow 0^{+}$transitions, ${ }^{38} \mathrm{Ca} \rightarrow{ }^{38 m} \mathrm{~K}$ and ${ }^{38 m} \mathrm{~K} \rightarrow{ }^{38} \mathrm{Ar}$, which provides a valuable constraint on the calculated isospin-symmetry-breaking corrections needed to derive $G_{V}$ from the experimental data. As anticipated by the Conserved Vector Current hypothesis, CVC, all fourteen transitions yield consistent values for $G_{\mathrm{V}}$. The value of $V_{u d}$ derived from their average makes it by far the most precisely known element of the CKM matrix, which, when combined with the other top-row elements, $V_{u s}$ and $V_{u b}$, leads to the most demanding test available of the unitarity of that matrix. Since CKM unitarity is a key pillar of the Electroweak Standard Model, this test is of fundamental significance.
\end{abstract}

\section{Introduction}

Superallowed beta decay between nuclear analogue states with $T=1$ and $J^{\pi}=0^{+}$occurs only via the vector current of the weak interaction: angular momentum conservation completely rules out the axial-vector current, which must carry off a spin of one and cannot connect two states that both have spin zero. Furthermore, since the parent and daughter states are analogues of one another, the strength of the transition is affected only by the small difference between the parent and daughter configurations resulting from isospin symmetry breaking, not by the dominant nuclear structure common to them both.

The measured strength of such a transition - expressed as an " $f t$ value" - can then be related directly to the vector coupling constant, $G_{\mathrm{v}}$ with the intervention of only a few small $(\sim 1 \%)$ calculated terms to account for radiative and isospin symmetry-breaking effects. Once $G_{\mathrm{V}}$ has been determined in this way, it is only another short step to obtain a value for $V_{u d}$, the up-down mixing element of the Cabibbo-Kobayashi-Maskawa (CKM) matrix, with which it is possible to test the top-row unitarity of that matrix. Since the unitary CKM matrix is a central pillar of the three-generation Standard Model, any experimentally determined deviation from CKM unitarity would be a signature of new physics beyond the Model; and even uncer-

\footnotetext{
a e-mail: hardy@comp.tamu.edu

be-mail: towner@comp.tamu.edu
}

tainty limits on a sum that agrees with unitarity can serve as a constraint on possible candidates for new physics.

Currently superallowed $\beta$-decay yields the most precise value for $V_{u d}$ and the most exacting test of CKM unitarity, with a precision of $0.06 \%$ on the latter $[1,2]$. This precision can be expected to improve further as a result of decay measurements that focus specifically on defining the effects of isospin-symmetry breaking between the analogue parent and daughter states in each superallowed transition. Of particular interest in this regard are mirror pairs of superallowed $0^{+} \rightarrow 0^{+}$transitions, the first one of which, ${ }^{38} \mathrm{Ca} \rightarrow{ }^{38 m} \mathrm{~K}$ and ${ }^{38 m} \mathrm{~K} \rightarrow{ }^{38} \mathrm{Ar}$, has only recently been completed [3]. Completion of such a pair requires an $f t$-value measurement of the previously uncharacterized superallowed transition from a $T_{z}=-1$ nucleus like ${ }^{38} \mathrm{Ca}$. In addition to this nucleus, three other candidates to complete a mirror pair are accessible to precision measurement: ${ }^{26} \mathrm{Si},{ }^{34} \mathrm{Ar}$ and ${ }^{42} \mathrm{Ti}$. With their likely addition in the near future, even more stringent constraints can be placed on the calculated isospin-symmentry-breaking correction terms, constraints that will in turn result in a reduced uncertainty on $V_{u d}$.

\section{Present status of superallowed decays}

In dealing with superallowed decays, it is convenient to combine some of the small correction terms with the measured $f t$-value and define a "corrected" $\mathcal{F} t$-value. Thus, 

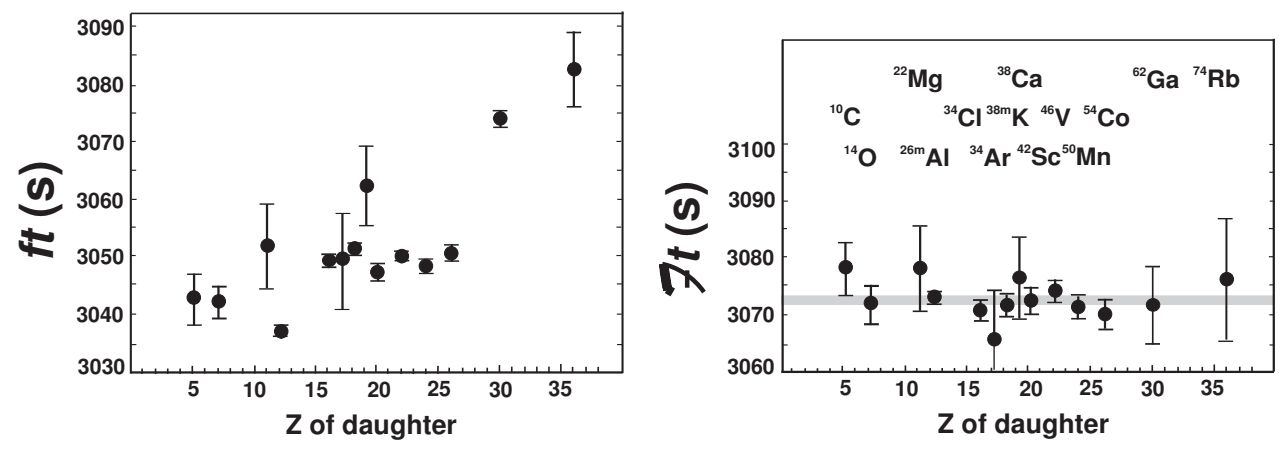

Figure 1. Results from the 2014 survey: uncorrected $f t$ values for the 14 best known superallowed decays on the left; the same results but incorporating the $\delta_{R}^{\prime}, \delta_{C}$ and $\delta_{N S}$ correction terms on the right. The grey band in the right panel is the average $\mathcal{F} t$ value and its uncertainty.

we write [1]

$$
\mathcal{F} t \equiv f t\left(1+\delta_{R}^{\prime}\right)\left(1+\delta_{N S}-\delta_{C}\right)=\frac{K}{2 G_{\mathrm{V}}^{2}\left(1+\Delta_{\mathrm{R}}^{\mathrm{V}}\right)},
$$

where $K=8120.2787(11) \times 10^{-10} \mathrm{GeV}^{-4} \mathrm{~s} ; \delta_{C}$ is the isospinsymmetry-breaking correction and $\Delta_{\mathrm{R}}^{\mathrm{V}}$ is the transitionindependent part of the radiative correction. The terms $\delta_{R}^{\prime}$ and $\delta_{N S}$ comprise the transition-dependent part of the radiative correction, the former being a function only of the electron's energy and the $Z$ of the daughter nucleus, while the latter, like $\delta_{C}$, depends in its evaluation on the details of nuclear structure. From this equation, it can be seen that a measurement of any one superallowed transition establishes an individual value for $G_{\mathrm{v}}$. A measurement of several of them tests the Conserved Vector Current (CVC) hypothesis that $G_{\mathrm{V}}$ is not renormalized in the nuclear medium. If indeed $G_{\mathrm{V}}$ is constant - i.e. all the $\mathcal{F} t$-values are the same - then an average value for $G_{\mathrm{V}}$ can be determined and $V_{u d}$ obtained from the relation $V_{u d}=G_{\mathrm{V}} / G_{\mathrm{F}}$, where $G_{\mathrm{F}}$ is the well known [4] weakinteraction constant for purely leptonic muon decay.

The $f t$-value that characterizes any $\beta$-transition depends on three measured quantities: the total transition energy, $Q_{E C}$, the half-life, $t_{1 / 2}$, of the parent state and the branching ratio, $R$, for the particular transition of interest. A new critical survey of world data on superallowed $0^{+} \rightarrow 0^{+}$beta decays has been completed and is currently being prepared for publication; it updates and will replace our previous survey, published five years ago [1]. The new survey lists $f t$ values for 14 transitions, which have been precisely determined from a very robust data set containing 222 individual measurements of comparable precision obtained from 177 published references. The results were then used to obtain the corrected $\mathcal{F} t$ values, with the outcome shown in Fig. 1.

It is immediately evident from the figure that the $\mathcal{F} t$ values are all consistent with one another from $A=10$ to $A=74$. This simultaneously confirms the CVC expectation of a constant value for $G_{\mathrm{V}}$ and demonstrates the absence of any significant scalar current, which would introduce an upward or downward curve into the $\mathcal{F} t$-value locus at low $Z$. It also goes a long way towards validat- ing the calculated isospin symmetry-breaking corrections: The calculations of $\delta_{C}$ and $\delta_{N S}$ for each transition in this analysis [5] employed the best available shell-model wave functions, which had been based on a wide range of spectroscopic data for nuclei in the same mass region. They were further tuned to agree with measured binding energies, charge radii and coefficients of the isobaric multiplet mass equation for the specific states involved. This means that the origins of these correction terms are completely independent of the superallowed decay data, so consistency in the corrected $\mathcal{F} t$ values gives powerful support to the calculated corrections used in the derivation of those $\mathcal{F} t$ values.

With a mutually consistent set of $\mathcal{F} t$ values, one is then justified in proceeding to determine the value of $G_{\mathrm{V}}$ and, from it, $V_{u d}$. The result we obtained from the new survey is, preliminarily, $V_{u d}=0.97417(21)$, which, when combined with Particle Data Group values for $V_{u s}$ and $V_{u b}$ [4], yields a CKM unitarity sum of $0.99978(55)$, in excellent agreement with Standard Model expectations. Note that this analysis was only possible because the $\mathcal{F} t$ values form a consistent set. Without demonstrated consistency with $\mathrm{CVC}$, there can be no justification for extracting a unique value for $G_{\mathrm{V}}$ from the data, let alone one for $V_{u d}$.

The uncertainty quoted for $V_{u d}$ is dominated by the theoretical uncertainty originating from $\Delta_{R}^{v}$, the so-called "inner" radiative correction. The uncertainties in the nuclear-structure-dependent corrections, $\delta_{C}$ and $\delta_{N S}$, are second in importance, while those from experiment rank a distant third. Although one might conclude from this observation that experiment has no further role to play in improving the CKM unitarity test, this would not be correct. Since the correction terms $\delta_{C}$ and $\delta_{N S}$ exhibit very pronounced differences from transition to transition (compare the two panels in Fig. 1, which differ principally by the application of these correction terms) their veracity can be tested and possibly improved by new measurements that either reduce the experimental uncertainties on the currently measured $f t$ values, or else increase the number of precisely measured transitions, particularly those that are mirror to currently known cases and those that have much larger calculated correction terms. Depending on whether 
these new results confirm the transition-to-transition variations obtained from the calculated corrections or not, the calculations may be validated or alternatively refined to restore agreement. Either way, it will likely be possible to reduce the associated theoretical uncertainties.

\section{Testing $\delta_{C}$ calculations}

In the past few years, a number of different groups have published $\delta_{C}$ values from calculations based upon a variety of different model approaches. Typically each calculation covers only a subset of the measured transitions but the subsets are not the same from calculation to calculation and, where overlap does exist, the results are not notably consistent with one another. This diversity of results has prompted us to develop a test [6] that allows each set of correction terms to be judged by its ability to produce a statistically consistent set of $\mathcal{F} t$ values (i.e. with $\chi^{2} / N \sim 1$ ) in agreement with the expectations of CVC. As part of our survey, we applied this test to all sets that cover at least half the number of well-measured superallowed transitions. The resultant $\chi^{2} / N$ values for the various calculations spanned a wide range, with only a single set yielding a value near one. In this way, we identified that set [5] as the one to use in our ultimate analysis of the experimental data (see Fig. 1)

There is a second test that can be expected to refine the selection process for $\delta_{C}$ calculations even further. It involves the measurement of mirror pairs of superallowed transitions, which has only become possible recently with the first case $-{ }^{38} \mathrm{Ca} \rightarrow{ }^{38 m} \mathrm{~K}$ and ${ }^{38 m} \mathrm{~K} \rightarrow{ }^{38} \mathrm{Ar}$ - appearing just six months ago [3]. This test also depends on the expected constancy of $\mathcal{F} t$ values, but in this instance it applies to the two members of a mirror pair of $0^{+} \rightarrow 0^{+}$transitions. Assuming these two $\mathcal{F} t$ values are the same, we can use Eq.(1) to write the ratio of experimental $f t$ values for a pair of mirror superallowed transitions as follows:

$$
\frac{f t^{a}}{f t^{b}}=1+\left(\delta_{R}^{\prime b}-\delta_{R}^{\prime a}\right)+\left(\delta_{N S}^{b}-\delta_{N S}^{a}\right)-\left(\delta_{C}^{b}-\delta_{C}^{a}\right),
$$

where superscript " $a$ " denotes the decay of the $T_{Z}=-1$ parent $\left({ }^{38} \mathrm{Ca} \rightarrow{ }^{38 m} \mathrm{~K}\right.$ in the case to be described here) and " $b$ " denotes the decay of the $T_{Z}=0$ parent $\left({ }^{38 m} \mathrm{~K} \rightarrow{ }^{38} \mathrm{Ar}\right)$. We illustrate the application of this test to two particular sets of $\delta_{C}$ calculations [6]. The first, denoted SM-WS, is the set that passed the $\chi^{2}$ test already described, while the second, SM-HF, was used in our previous survey to establish a systematic uncertainty. The advantage offered by Eq. (2) is that in these models the (theoretical) uncertainty on a difference term such as $\left(\delta_{C}^{b}-\delta_{C}^{a}\right)$ is significantly less than the uncertainties on $\delta_{C}^{b}$ and $\delta_{C}^{a}$ individually.

To understand this, one must first recognize how $\delta_{C}$ and its quoted uncertainty were derived in the first place [5]. The term itself was broken down into two components, $\delta_{C 1}$ and $\delta_{C 2}$, with the first corresponding to a finitesized shell-model (SM) calculation typically restricted to one major shell, while the second took account of configurations outside that model space via a calculation of the mismatch between the parent and daughter radial wave

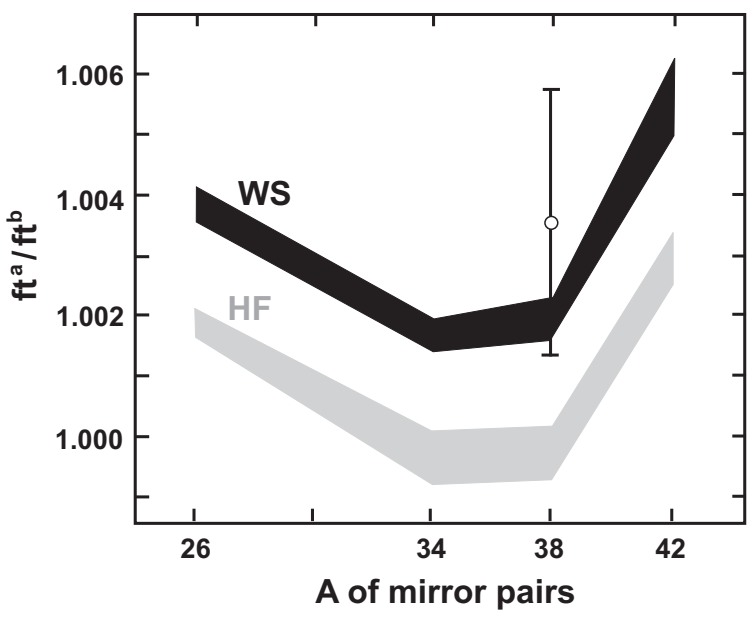

Figure 2. Mirror-pair $f t^{a} / f t^{b}$ values for $A=26,34,38$ and 42 , the four cases currently accessible to high-precision experiment. The black and grey bands connect calculated results that utilize Woods-Saxon (WS) and Hartree-Fock (HF) radial wave functions, respectively. Our measured result for the $A=38 \mathrm{mir}-$ ror pair is shown as the open circle with error bars.

functions. The parameters used for the shell-model calculation were taken from the literature, where they had been based on a wide range of independent spectroscopic data from nearby nuclei. In all cases, more than one parameter set was available, so more than one calculated value was obtained for each correction term. The value adopted for $\delta_{C 1}$ was then the average of the results obtained from the different parameter sets, and the quoted "statistical" uncertainty reflected the scatter in those results. If the same approach is used to derive the mirror differences of correction terms $\left(\delta_{C 1}^{b}-\delta_{C 1}^{a}\right)$, the scatter among the results from different parameter sets is less than the scatter in either $\delta_{C 1}^{b}$ or $\delta_{C 1}^{a}$.

For $\delta_{C 2}$ there is a further source of theoretical uncertainty that arises from the choice of potential used to obtain the parent and daughter radial wave functions. Both Woods-Saxon (WS) and Hartree-Fock (HF) eigenfunctions have been used but there is a consistent difference between their results. Consequently in our previous survey [1] a "systematic" uncertainty corresponding to half the difference was assigned to $\delta_{C 2}$, which naturally increased the uncertainty on the derived $V_{u d}$ and on the unitarity sum.

With the statistical (theoretical) uncertainty contribution from $\delta_{C}$ reduced in the mirror $f t$-value ratio, Eq. (2) offers the opportunity to use experiment to distinguish cleanly between WS and HF radial wave functions. If one set of calculations were to be convincingly eliminated, then the systematic uncertainty on $\delta_{C}$ could also be eliminated and the uncertainty in $V_{u d}$ reduced.

With current capabilities for producing superallowed $T_{Z}=-1$ parent nuclei in sufficient quantity for a highstatistics measurement, there are three mirror pairs in addition to the one at $A=38$ that can be completed. These are ${ }^{26} \mathrm{Si} \rightarrow{ }^{26 m} \mathrm{Al}$ and ${ }^{26 m} \mathrm{Al} \rightarrow{ }^{26} \mathrm{Mg} ;{ }^{34} \mathrm{Ar} \rightarrow{ }^{34} \mathrm{Cl}$ and ${ }^{34} \mathrm{Cl} \rightarrow{ }^{34} \mathrm{~S}$; and ${ }^{42} \mathrm{Ti} \rightarrow{ }^{42} \mathrm{Sc}$ and ${ }^{42} \mathrm{Sc} \rightarrow{ }^{42} \mathrm{Ca}$. The calcu- 
lated values of $f t^{a} / f t^{b}$ for all four are plotted in Fig. 2, where it can be seen that they are cleanly separated from one another. Though the actual differences between them are small, they are large enough for experiment to be capable of selecting one calculation over the other.

\section{Measurement of the ${ }^{38} \mathrm{Ca}$ branching ratio}

At Texas A\&M, we are currently embarked on a program to measure $T_{z}=-1$ parent superallowed decays, particularly those of ${ }^{26} \mathrm{Si},{ }^{34} \mathrm{Ar},{ }^{38} \mathrm{Ca}$ and ${ }^{42} \mathrm{Ti}$, which complete mirror pairs. We have already published our half-life results for the first three of these cases [7-9] and now have measured the branching ratio for the ${ }^{38} \mathrm{Ca}$ decay [3], the last link required to complete the $A=38$ mirror pair.

We produced $444-\mathrm{ms}{ }^{38} \mathrm{Ca}$ using a $30 A-\mathrm{MeV}^{39} \mathrm{~K}$ primary beam from the Texas A\&M K500 superconducting cyclotron to initiate the ${ }^{1} \mathrm{H}\left({ }^{39} \mathrm{~K}, 2 n\right){ }^{38} \mathrm{Ca}$ reaction on a $\mathrm{LN}_{2}$-cooled hydrogen gas target. The fully stripped ejectiles were separated by their charge-to-mass ratio, $q / m$, in the MARS recoil separator, producing a ${ }^{38} \mathrm{Ca}$ beam at the focal plane, where the beam composition was monitored by the periodic insertion of a position-sensitive silicon detector. With the detector removed, the ${ }^{38} \mathrm{Ca}$ beam exited the vacuum system through a $50-\mu$ m-thick Kapton window, passed successively through a $0.3-\mathrm{mm}$-thick BC404 scintillator and a stack of aluminum degraders, finally stopping in the $76-\mu \mathrm{m}$-thick aluminized Mylar tape of a fast tape-transport system. The combination of $q / m$ selectivity in MARS and range separation in the degraders provided implanted samples that were $99.7 \%$ pure ${ }^{38} \mathrm{Ca}$. Approximately 24,000 atoms/s of ${ }^{38} \mathrm{Ca}$ were implanted in the tape.

During the measurement, each ${ }^{38} \mathrm{Ca}$ sample was accumulated in the tape for $1.6 \mathrm{~s}$, with its rate of accumulation being measured by the scintillation detector located ahead of the degrader stack. Then the beam was turned off and the tape moved the sample in $200 \mathrm{~ms}$ to a shielded counting location $90 \mathrm{~cm}$ away, where data were collected for $1.54 \mathrm{~s}$, after which the cycle was repeated. This computercontrolled sequence was repeated continuously for nearly 5 days.

At the counting location, the sample was positioned precisely between a 1-mm-thick BC-404 scintillator to detect $\beta^{+}$particles, and our specially calibrated $[10,11] 70 \%$ HPGe detector for $\gamma$ rays. The former was located $3 \mathrm{~mm}$ from one side of the tape, while the latter was $15.1 \mathrm{~cm}$ away on the other side. We saved $\beta$ - $\gamma$ coincidences eventby-event, recording the energy of each $\beta$ and $\gamma$ ray, the time difference between their arrival, and the time that the event itself occurred after the beginning of the counting period. For each cycle we also recorded the rate of accumulation of ${ }^{38} \mathrm{Ca}$ ions in the tape as a function of time, the total number of $\beta$ - and $\gamma$-ray singles, and the output from a laser ranging device that recorded the distance of the stopped tape from the HPGe detector. From cycle to cycle that distance could change by a few tenths of a millimeter, enough to require a small adjustment to the HPGe detector efficiency. Our recorded spectrum of $\beta$-coincident $\gamma$ rays appears in Fig 3 .

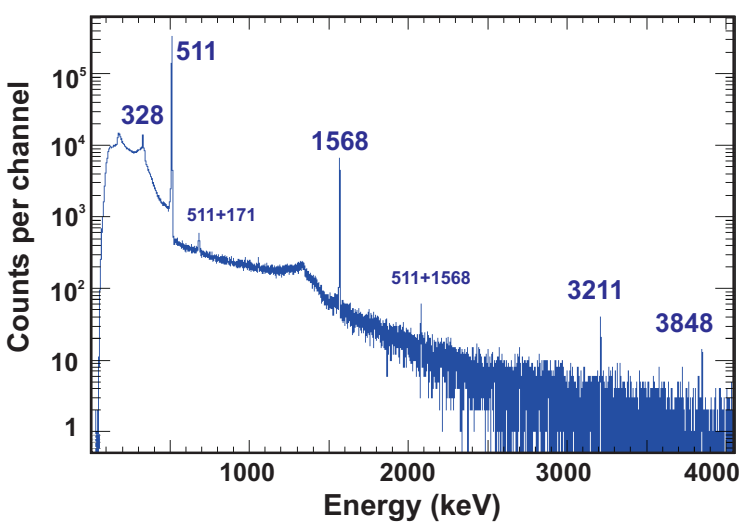

Figure 3. Spectrum of $\gamma$ rays observed in prompt coincidence with positrons from the decay of ${ }^{38} \mathrm{Ca}$. The small peak labeled " $511+171$ " is caused by positron annihilation, from which one $511-\mathrm{keV} \gamma$ ray sums with a back-scattered $\gamma$ ray from the second 511-keV $\gamma$ ray. The " $511+1568$ " peak is the result of coincidence summing between a $1568-\mathrm{keV} \gamma$ ray and annihilation radiation from the positron decay that preceded it.

All $\beta$ transitions from ${ }^{38} \mathrm{Ca}$ populate prompt- $\gamma$ emitting levels in ${ }^{38} \mathrm{~K}$, except for the superallowed branch. To obtain the superallowed branching ratio, our approach was first to determine the number of $1568-\mathrm{keV} \gamma$ rays relative to the total number of positrons emitted from ${ }^{38} \mathrm{Ca}$. This established the $\beta$-branching ratio to the $1^{+}$state in ${ }^{38} \mathrm{~K}$ at $1698 \mathrm{keV}$. Next, from the relative intensities of all the other (weaker) observed $\gamma$-ray peaks, we determined the total Gamow-Teller $\beta$-branching to all $1^{+}$states. Finally, by subtracting this total from $100 \%$, we arrived at the branching ratio for the superallowed transition to the $0^{+}$isomeric state ${ }^{38 m} \mathrm{~K}$.

More specifically, if the $\gamma$ ray de-exciting state $i$ in ${ }^{38} \mathrm{~K}$ is denoted by $\gamma_{i}$, then the $\beta$-branching ratio, $R_{i}$, for the $\beta$ transition populating that state can be written:

$$
R_{i}=\frac{N_{\beta \gamma_{i}}}{N_{\beta} \epsilon_{\gamma_{i}}} \frac{\epsilon_{\beta}}{\epsilon_{\beta_{i}}}
$$

where $N_{\beta \gamma_{i}}$ is the total number of $\beta$ - $\gamma$ coincidences in the $\gamma_{i}$ peak; $N_{\beta}$ is the total number of beta singles corresponding to ${ }^{38} \mathrm{Ca} \beta$ decay; $\epsilon_{\gamma_{i}}$ is the efficiency of the HPGe detector for detecting $\gamma_{i} ; \epsilon_{\beta_{i}}$ is the efficiency of the plastic scintillator for detecting the betas that populate state $i$; and $\epsilon_{\beta}$ is the average efficiency for detecting the betas from all ${ }^{38} \mathrm{Ca}$ transitions.

After accounting for dead-time, pile-up, coincidence summing and other small effects, we obtained a superallowed branching ratio of $0.7728 \pm 0.0014_{\text {stat }} \pm 0.0009_{\text {syst }}$ or, with the uncertainties combined in quadrature, $0.7728 \pm$ 0.0016 . From our recent survey, the half-life of ${ }^{38} \mathrm{Ca}$ is $443.77(35) \mathrm{ms}$ and the $Q_{E C}$ value for its superallowed branch is $6612.12(7) \mathrm{keV}$. Taking these results with our new value for the branching ratio and correcting for electron capture, we arrive at an $f t$ value for the ${ }^{38} \mathrm{Ca}$ superallowed branch of $f t^{a}=3062.3(68) \mathrm{s}$. The $f t$ value for the mirror transition from ${ }^{38 m} \mathrm{~K}$ is $f t^{b}=3051.45(92) \mathrm{s}$. The 
ratio of the two, $f t^{a} / f t^{b}=1.0036(22)$, appears in Fig. 2, where it can be compared with the two competing calculations.

Although our experimental result favors the SM-WS calculation, it is not yet definitive. The final verdict must await measurements of the other three mirror pairs, especially the one with $A=34$. Only a precise branching ratio for ${ }^{34} \mathrm{Ar}$ is missing for that pair and it promises to have a tighter uncertainty than can be achieved for the other $T_{Z}=-1$ parent decays. In the meantime, however, the combination of this result together with the $\chi^{2}$ test described at the beginning of section 3 strongly disfavors the SM-HF calculation so, in our recent survey, we use only the SMWS $\delta_{C}$ values and no longer employ SM-HF to determine a systematic shift and uncertainty.

\section{Conclusion}

Already, superallowed $0^{+} \rightarrow 0^{+}$beta decay produces a very precise value for $V_{u d}$ and enables a demanding test of CKM unitarity. Although any significant reduction of the current uncertainty must await a more precise calculation of the $\Delta_{\mathrm{R}}^{\mathrm{V}}$ radiative correction, some modest improvement can still be achieved by reducing the uncertainty on the nucleus-dependent corrections $\delta_{C}$ and $\delta_{N S}$ through experiments designed to test these corrections. Of greatest potential at present are measurements that complete mirror pairs of superallowed transitions. It is anticipated that the ${ }^{38} \mathrm{Ca}$ measurement described here will be followed in due course by similar measurements on ${ }^{26} \mathrm{Si},{ }^{34} \mathrm{Ar}$ and ${ }^{42} \mathrm{Ti}$. Together they will add significantly to the constraints that can be applied to the nucleus-dependent correction terms.

\section{Acknowledgements}

This material is based upon work supported by the U.S. Department of Energy, Office of Science, Office of Nuclear Physics, under Award Number DE-FG0393ER40773, and by the Robert A. Welch Foundation under Grant No. A-1397.

\section{References}

[1] J.C. Hardy and I.S. Towner, Phys. Rev. C 79, 055502 (2009)

[2] J.C. Hardy and I.S. Towner, Ann. Phys. (Berlin) 525, 443 (2013)

[3] H.I. Park, J.C. Hardy, V.E. Iacob, M. Bencomo, L. Chen, V. Horvat, N. Nica, B.T. Roeder, E. Simmons, R.E. Tribble and I.S. Towner, Phys. Rev. Lett. 112, 102502 (2014)

[4] K.A. Olive et al. (Particle Data Group), Chin. Phys. 38, 090001 (2014)

[5] I.S. Towner and J.C. Hardy, Phys. Rev. C 77, 025501 (2008)

[6] I.S. Towner and J.C. Hardy, Phys. Rev. C 82, 065501 (2010)

[7] V.E. Iacob, J.C. Hardy, J.F. Brinkley, C.A. Gagliardi, V.E. Mayes, N. Nica, M. Sanchez-Vega, G. Tabacaru, L. Trache and R.E. Tribble, Phys. Rev. C 74, 055502 (2006)

[8] V.E. Iacob, J.C. Hardy, A. Banu, L. Chen, V.V. Golovko, J. Goodwin, V. Horvat, N. Nica, H.I. Park, L. Trache and R.E. Tribble, Phys. Rev. C 82, 035502 (2010)

[9] H.I. Park, J.C. Hardy, V. E. Iacob, A.Banu, L. Chen, V.V. Golovko, J. Goodwin, V. Horvat, N. Nica, E. Simmons, L. Trache and R.E. Tribble, Phys. Rev. C 84, 065502 (2011)

[10] R.G. Helmer, J.C. Hardy, V.E. Iacob, M. SanchezVega, R.G. Neilson and J. Nelson, Nucl. Instrum. Methods Phys. Res. Sect. A 511, 360 (2003)

[11] R.G. Helmer, N. Nica, J.C. Hardy and V.E. Iacob, Appl. Rad. Isot. 60, 173 (2004) 
